\title{
Surgeons' Attitudes Are Associated With Reoperation and Readmission Rates
}

\author{
John Kadzielski MD, Frank McCormick MD, \\ James H. Herndon MD, MBA, Harry Rubash MD, \\ David Ring MD, PhD
}

Published online: 30 May 2014

(C) The Association of Bone and Joint Surgeons $(2014$

\begin{abstract}
Background Attitudes influence how people make decisions. In an effort to decrease pilot judgment-related accidents, the Federal Aviation Administration teaches new pilots about hazardous attitudes that are believed to be incompatible with safe flight: macho, impulsive, worry, resignation, self-confidence, and antiauthority. If these attitudes are hazardous for pilots and their passengers, they may also be incompatible with the reliable and safe delivery of surgical care.

Questions/purposes The purposes of this study were (1) to ascertain to what extent surgeons harbor hazardous attitudes; and (2) to determine their relationship, if any, to reoperation and readmission rates.
\end{abstract}

The presentation of this work has been supported by the

Massachusetts General Hospital Department of Orthopaedic Surgery 2009 Academic Enrichment Fund Grant as well as the Partners Center of Expertise in Quality and Patient Safety Scholarship (JK, FM, HR). All ICMJE Conflict of Interest Forms for authors and Clinical Orthopaedics and Related Research ${ }^{\mathbb{R}}$ editors and board members are on file with the publication and can be viewed on request.

Each author certifies that his or her institution approved the human protocol for this investigation, that all investigations were conducted in conformity with ethical principles of research, and that informed consent for participation in the study was obtained.

This work was performed at Massachusetts General Hospital, Boston, MA, USA.

Electronic supplementary material The online version of this article (doi:10.1007/s11999-014-3687-6) contains supplementary material, which is available to authorized users.

J. Kadzielski ( $₫)$

Hand and Upper Extremity Surgery, South Shore Orthopedics,

2 Pond Park Road, Suite 102, Hingham, MA 02043, USA

e-mail: jkadzielski@partners.org

F. McCormick

Holy Cross Orthopedic Institute, Ft Lauderdale, FL, USA
Methods We selected validated aviation psychology tools that are used to measure these attitudes in pilots. We converted the aviation scenarios to analogous situations for surgeons and invited all surgeons from one academic program to participate in this study. A total of 41 surgeons were eligible to participate; 37 (90\%) completed the attitude prevalence protocol and $31(76 \%)$ had complete reoperation and readmission data for the correlation and regression analysis. Attending orthopaedic surgeons completed the Modified Surgeon Hazardous Attitude Scale as well as a series of additional instruments.

Results Levels of macho thought to be hazardous in pilots were present in nine (24\%) surgeons. Similar, elevated levels of self-confidence were found in three (8\%) surgeons. High levels of impulsivity were found in 5\% (two surgeons) and high levels of antiauthority were found in 3\% (one surgeon). Only one (3\%) surgeon reported elevated levels of worry and no surgeon reported hazardous levels of resignation. Thirty percent (11 surgeons) of surgeons harbored at least one elevated attitude level. In a regression model, macho attitude levels predicted $19 \%$ of the variation in surgeons' rate of readmissions and reoperations.

Conclusions High levels of hazardous attitudes may not be consistent with the routine delivery of safe surgical care in a teamwork setting where human factors and safe systems are the key to success. Further research is needed to determine if abnormally high levels of these hazardous attitudes impact patient care.

\section{J. H. Herndon, D. Ring}

Hand and Upper Extremity Service, Department of Orthopaedic

Surgery, Massachusetts General Hospital, Boston, MA, USA

\section{H. Rubash}

Arthroplasty Service, Department of Orthopaedic Surgery,

Massachusetts General Hospital, Boston, MA, USA 
Level of Evidence Level II, prognostic study. See Guidelines for Authors for a complete description of levels of evidence.

\section{Introduction}

Since the advent of the jet age, attitudes have been linked to aviation accidents. General Chuck Yeager in his autobiography stated, "Arrogance got more pilots in trouble than faulty equipment" [28]. However, to say that certain attitudes directly lead to specific behaviors and outcomes may be overstating their influence. Nevertheless, it is generally believed that an attitude-a "personal motivational predisposition to respond to persons, situations, or events in a given manner"-does influence how a person makes decisions [5]. The Federal Aviation Administration (FAA) reported that over half of general aviation accidents are related to pilot decision-making [4]. In a 1984 joint effort to evaluate the effectiveness of pilot judgment training between the FAA and the Canadian Air Transportation Administration, researchers identified five hazardous attitudes that were thought to adversely influence judgmentmacho, impulsive, antiauthority, resignation, and invulnerable [3]. In 1987, the FAA began teaching new pilots about these five hazards. In a validation study on an analogous population of college-aged drivers, a sixth attitude emerged, self-confidence [12]. The FAA acknowledged this validation work done on drivers and has continued to educate all pilotsin-training about hazardous attitudes [6]. Part of that training includes mitigation techniques and the consideration of antidotes (Fig. 1) [4-6, 12, 16].

Although widely accepted for the last 20 years in aviation, the idea that a hazardous attitude may contribute to an undesirable outcome is still largely foreign to the surgical world. The identification of context-independent hazardous attitudes

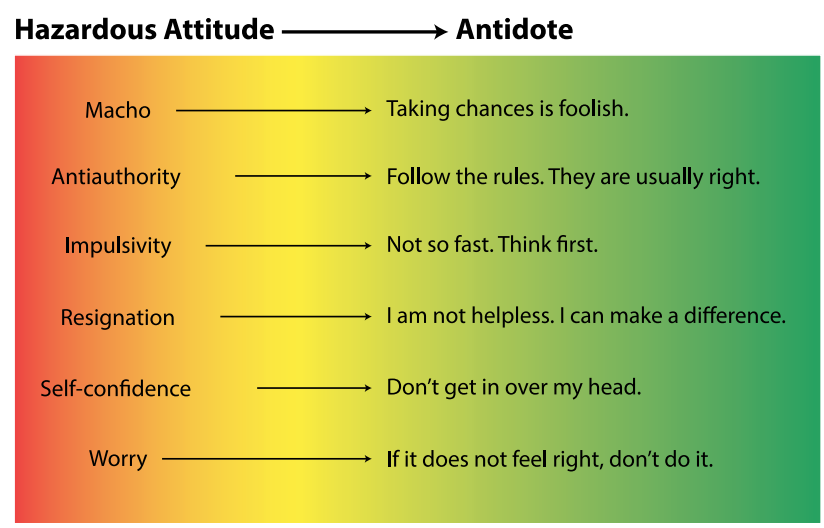

Fig. 1 This figure depicts the six hazardous attitudes and their proposed antidotes $[6,12,16]$. Reprinted with permission from Nicole Wolf. in surgeons and determination of their relationship with complications has implications for patient safety, quality, and surgical training. Doctors and surgeons may have higher levels of hazardous attitudes than other populations. To explain an increasing trend of general aviation accidents in people with advanced degrees, eg, MD or JD [27], one researcher suggested that such people have more predisposing personality factors including aggression, independence, and self-sufficiency [2]. Furthermore, like gamblers, surgeons who elect higher-risk cases with uncertain outcomes may learn differently from the general population receiving a disproportionally larger reward for success but only internalizing a small negative sensation from an undesirable outcome [8]. Although these findings have not been substantiated in a surgeon population, if they are true, they point to the importance of this current investigation.

The primary purpose of this study is to determine the prevalence and types of hazardous attitudes harbored by orthopaedic surgeons. The secondary aim of this study is to determine if there is an association between surgeons' hazardous attitudes and serious complications as represented by hospital readmission and reoperation rates.

\section{Materials and Methods}

\section{Subject Selection}

Attending orthopaedic surgeons $(n=46)$ in the Department of Orthopaedic Surgery at Massachusetts General Hospital were invited to participate. Attending surgeons who were members of the study staff were excluded given that their responses could be biased $(n=3)$. Surgeons who no longer had patient encounters were likewise excluded $(n=2)$. Forty-one surgeons were eligible for participation. Three surgeons did not respond to repeated invitations to participate and one surgeon declined participation. Of note, these last four surgeons were of the same subspecialty, which was not disclosed in the article because the cohort is so small that they would become identifiable as individuals. Thirty-seven of 41 surgeons $(90 \%)$ provided data for attitude prevalence analysis. Roughly $19 \%$ of the sample population reported their specialty as sports $(n=7$; Table 1). Average years in practice for our cohort were $17 \pm 12$. Surgeons who no longer operated were included in the questionnaire component of this protocol but excluded from the reoperation and readmission analysis $(\mathrm{n}=3)$. Surgeons who had incomplete reoperation and readmission data because they started during $(n=2)$ or retired $(n=1)$ during the study period were also excluded from the readmission and reoperation analysis. A total of 31 surgeons $(76 \%)$ were included in the reoperation and readmission correlation and regression analysis. 
Table 1. The number of surgeons in each orthopaedic subspecialty

\begin{tabular}{lcc}
\hline Subspecialty & $\begin{array}{l}\text { Number of } \\
\text { surgeons }\end{array}$ & $\begin{array}{c}\text { Percent of } \\
\text { department }\end{array}$ \\
\hline Arthroplasty & 4 & 10.8 \\
Foot and ankle & 4 & 10.8 \\
Hand and upper extremity & 3 & 8.1 \\
General orthopaedics & 2 & 5.4 \\
Oncology & 4 & 10.8 \\
Pediatrics & 2 & 5.4 \\
Shoulder & 3 & 8.1 \\
Spine & 5 & 13.5 \\
Sports & 7 & 18.9 \\
Trauma & 3 & 8.1 \\
Total & 37 & 100.0 \\
\hline
\end{tabular}

\section{Subject Enrollment}

Attending surgeons were invited to participate in the institutional review board-approved protocol at a staff meeting and contacted by email if they were absent. Consent was obtained by a trained study staff member.

\section{Confidentiality Procedures}

After informed consent was obtained, surgeons received an email invitation to participate through a secure email server. The email contained a randomly generated participant number (Microsoft ${ }^{\mathbb{R}}$ Excel $^{\mathbb{R}}$ randomize function; Microsoft Inc, Redmond, WA, USA). The data linking surgeons to their subject number were kept on a password-protected secure network drive approved by the institutional review board. Surgeons completed an Secure Sockets Layer (SSL) encrypted online questionnaire using their randomly generated subject number (www.surveymonkey.com). Data on the commercial service were encrypted.

\section{Measures}

\section{Demographic Data}

Standard demographic data were collected including sex, age, years in practice, subspecialty, and fellowship training.

\section{Reoperation and Readmission Data}

Currently there is no valid measure of total complications available to researchers. We did have access to accurate 30-day reoperation and readmission data from the Massachusetts
General Hospital Quality and Safety Division. It was decided by consensus among the authors that readmission and reoperation rates would catch many of the serious complications, acknowledging that these are likely to capture only a small percentage of overall complications. Accepting this limitation, surgeon-specific reoperation and readmission data for 2009 and 2010 were recorded and coded such that they could be identified by the surgeons' randomly generated participant number. Surgeons were also asked to report what percentage of their complications that they had treated in patients as outpatients so researchers could estimate how many overall complications might be available for future study.

\section{Modified Surgeon Hazardous Attitude Scale}

The Hazardous Attitude Scale has been modified many times since its inception $[1,4,5,11,18]$ and is currently a 30-question factor-weighted questionnaire, which is freely available online to pilots and to the general public on a web site entitled Aviation Human Factors: Improving General Aviation Training Through Research and Training [17]. The average score for each attitude among pilots is 15 and scores above 20 are considered hazardous [17]. The context of the questions was shifted from aviation-based to surgically based and the scoring was unchanged. See Appendix 1 (Supplemental materials are available with the online version of $\mathrm{CORR}^{\circledR}$.) for the surgical converted hazardous attitude scales.

\section{Modified Surgeon Safety Locus of Control Scale}

The concept of a safety locus of control was originally described in 1985 by Jones and Wuebker [19]. The concept refers to whom people believe is in control of safety in their environment, eg, internal (referring to self) or external (referring to others). Jones and Wuebker later validated their scale focusing on occupational accidents with healthcare workers, a population not defined but estimated to be $80 \%$ nurses [20]. Safety locus of control was modified and validated for use in aviation by Hunter in 2002 [14]. The mean pilot score was $38.8 \pm 4.3$ [14]. Hunter reported that higher internal locus of controls was preferable overall and suggested that pilots with an internal locus of control score $<30$ were at risk for a hazardous flight event or accident [15]. Hunter also noted that as pilots gained more experience, they reported more internal and less external locus of control [14]. The Aviation Safety Locus of Control Scale, which is available online to pilots and the general public, was modified to reflect surgeons rather than pilots [15]. The Modified Surgeon Safety Locus of Control Scale is located in Appendix 2. 


\section{Modified Surgeon Attitude Scale}

In 1999, researchers validated a scale used to identify attitudes toward unsafe acts in Australian general aviation pilots [24]. This 25-question scale showed that pilots who had higher levels of unsafe attitudes had significantly more human error-related accidents or incidents [24]. The mean for Australian pilots involved in an accident was 86 and the mean for pilots not involved in an accident was 79 (analysis of covariance $\mathrm{F}=4.08, \mathrm{p}<0.05$ ). Scores were calculated using a 5-point Likert scale with 1 being strongly disagree and 5 being strongly agree. The score is a sum of each response. Using the same methodology as with the Modified Hazardous Attitude Scale, the Australian Aviation Attitude Scale was modified to establish surgical relevance. The Modified Surgeon Attitude Scale is in Appendix 3.

\section{Modified Safety Climate Questionnaire}

Surgeons completed the Modified Patient Safety Climate Questionnaire [22]. This questionnaire is largely based on the Patient Safety Cultures in Healthcare Organizations instrument [9]. Outcomes of this study are reported as rates of "problematic responses," responses that implied an absence of a safety climate. Neutral responses were not reported as problematic. In previous research, hospital personnel reported to be "mostly nurses and physicians" in high-risk roles (operating room, emergency department, intensive care unit, pediatric intensive care unit) reported an average of $20.9 \%$ problematic responses as compared with $5.6 \%$ by naval aviators [9, 10, 21, 25, 26]. Although not independently validated, researchers selected the Modified Safety Climate Questionnaire for this study because it has been used to describe the safety climate among the residents in this hospital in 2004, 2005, 2008, and 2009 who reported an average overall all problematic response rate of $12.6 \%$ and would yield meaningful data for comparison [22]. Items with a $>10 \%$ problematic response rate were considered a problem that needed to be addressed by author consensus in our previous article [22].

\section{Statistical Analysis}

Surgeons completed the questionnaire in 2010. Reoperation and readmission data were acquired through the Massachusetts General Hospital Quality and Safety Division from 2009 and 2010. Descriptive statistics were used to describe the surgeons' demographics and responses. Pearson chisquare tests were used to determine if there were any significant differences in complications and attitudes between subspecialties. To determine if surgeons' responses had a linear relationship with reoperations and readmissions,
Pearson's correlations were calculated. Factors with significant correlations were entered as independent variables into a linear regression model to determine which factors could predict the variation in reoperation and readmission rates between surgeons. Given that sample size was limited by department size, a power analysis was not carried out.

\section{Results}

Prevalence and Types of Hazardous Attitudes

Levels of attitudes thought to be hazardous in pilots were identified in our surgeon population. The Modified Surgeon Hazardous Attitude Scale identified elevated levels of macho in $24 \%$ of surgeons (nine of 37). Elevated levels of self-confidence were found in $8 \%$ of surgeons (three of 37). High levels of impulsivity were found in 5\% of surgeons (two of 37) and abnormally high levels of antiauthority were found in 3\% of surgeons (one of 37). Only 3\% of surgeons (one of 37) reported high levels of worry and no surgeon reported hazardous levels of resignation. Thirty percent of surgeons (11 of 37) harbored at least one abnormally high hazardous attitude (Fig. 2). On the

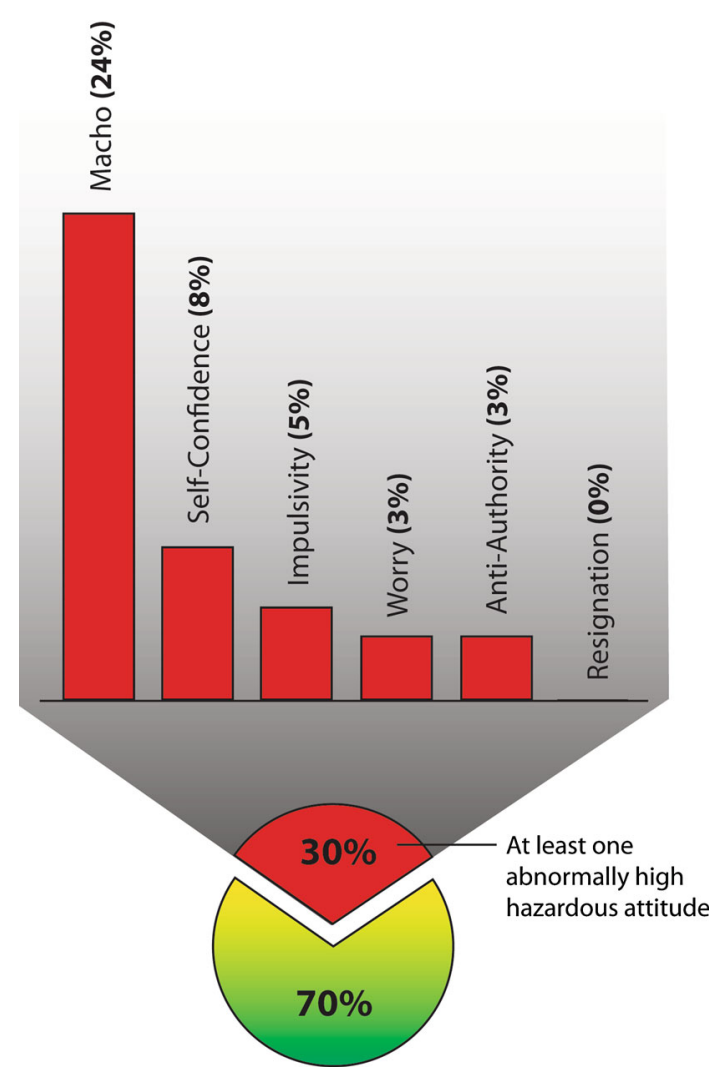

Fig. 2 This figure depicts the percentage of surgeons with dangerously high levels of each of the hazardous attitudes. Reprinted with permission from Nicole Wolf. 
Modified Surgeon Safety Locus of Control Scale, $32 \%$ of surgeons (12 of 37) scored below a 30, the cutoff score used in aviation to identify pilots who are at increased risk of an accident.

The Modified Surgeon Attitude Scale identified 5\% of surgeons (two of 37) who scored higher than 86 , which was the mean score of the group of pilots who had been involved in an accident. Sixteen percent of surgeons (six of 37) scored below 86 but above 79 , the mean of the group of pilots who did not have an accident.

When safety climate was assessed, surgeons reported a $16.2 \%$ overall mean problematic response rate. Problematic response rates ranged from $0 \%$ to $62.5 \%$ on individual questions among this group of surgeons. The rate of problematic responses did not vary among subspecialties $\left(\chi^{2}=82.31, \mathrm{p}=0.89\right)$. The surgeons report management's ability to reach well to unexpected changes and loss of experienced personnel as key problems that threatened the safety climate (Table 2).

\section{Associations Among Attitudes, Other Factors, and Readmissions}

Macho attitude $(r=0.436, p=0.014)$ and choice of subspecialty $(r=0.354, p=0.05)$ moderately correlated with the total number of readmissions and reoperations. There were no correlations between complications and self-confidence, impulsivity, antiauthority, resignation, internal safety locus of control, external safety locus of control, Modified Surgeon Attitude Scale, and the safety climate problematic response rate (all $\mathrm{p}>0.087$ ). Years in practice did not correlate with total number of readmissions and reoperations (Pearson $\mathrm{r}=-0.26, \mathrm{p}=0.88$ ).

When the significant factors from the correlation were entered into a regression model as independent variables, only macho attitude $(\beta=4.315, \quad p=0.014$, coefficient $=-55.309$ ) was identified as a predictor, accounting for $19.0 \%\left(\mathrm{R}^{2}=0.19\right)$ of the variance in reoperation and readmission rates $(\mathrm{F}=6.817, \mathrm{p}=0.014)$. As macho attitude levels increased, so did the numbers of readmissions and reoperations (Fig. 3). The trauma, oncology, and spine services had the highest reoperation and readmission rates over the 2-year collection period (Table 3). Sports had the lowest readmissions and reoperations during the 2-year data collection period. Collectively, there was no difference in readmission and reoperation rates among services $\left(\chi^{2}=224.191, \mathrm{p}=0.098\right)$. Fellowship training did not correlate with total number of readmissions and reoperations $(\mathrm{r}=0.161, \mathrm{p}=0.342)$.

Trauma, oncology, and spine surgeons had the highest mean annual readmission and reoperation rates per surgeon (Table 4). Additionally, given that readmission and

Table 2. The top 10 safety climate issues with the highest levels of problematic responses

Safety climate: top 10 items with the highest problematic response rates unexpected changes to its plan

2 Loss of experienced personnel has negatively affected my ability to provide high-quality patient care

3 Senior management does not hesitate to temporarily restrict clinicians who are under high personal stress

4 I am provided adequate resources (personnel, budget, and equipment) to provide safe patient care

5 In my department, there is significant peer pressure to discourage unsafe patient care

6 Individuals in my program are willing to report behavior that is unsafe for patient care

7 People in leadership positions set the example for compliance with policies and procedures that promote safe patient care

8 My program uniformly prescribes performance $\quad 14.3$ standards to ensure patient safety

9 My program follows a specific process to review performance standards to ensure patient safety

10 Patient safety decisions are made at the proper levels by the most qualified people

60.0
Percentage of problematic responses*

* Overall mean problematic response rate for residents in the same program who worked with these surgeons was $12.6 \%$ [27]. Overall mean problematic response rate for naval aviators was $5.6 \%$ [28].

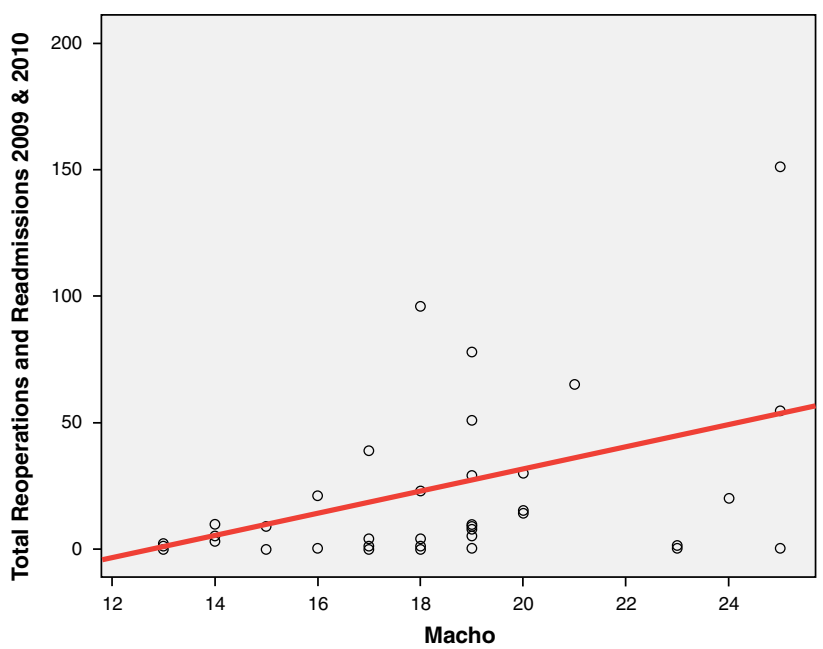

Fig. 3 This graph depicts the linear relationship between reoperation and readmission rates and macho attitude. The regression showed that macho, as an independent variable, accounted for $19 \%$ of the variance in reoperation and readmission rates. Reprinted with permission from Nicole Wolf. 
Table 3. Mean and total sum of reoperations and readmission from 2009 and 2010*

\begin{tabular}{|c|c|c|c|c|}
\hline Subspecialty & $\begin{array}{l}\text { Sum of readmissions } \\
\text { and reoperations } \\
2009 \text { and } 2010\end{array}$ & $\begin{array}{l}\text { Number of } \\
\text { surgeons }\end{array}$ & $\begin{array}{l}\text { Mean readmissions } \\
\text { and reoperations per } \\
\text { surgeon } 2009 \text { and } 2010\end{array}$ & SD \\
\hline Arthroplasty & 42 & 3 & 14.0 & 11.3 \\
\hline Foot and ankle & 18 & 3 & 6.0 & 3.5 \\
\hline Hand and upper extremity & 29 & 3 & 9.7 & 5.0 \\
\hline General orthopaedics & 2 & 1 & 2.0 & $\$$ \\
\hline Oncology & 159 & 3 & 53.0 & 13.1 \\
\hline Pediatrics & 9 & 2 & 4.5 & 6.4 \\
\hline Shoulder & 24 & 3 & 8.0 & 7.2 \\
\hline Spine & 138 & 5 & 27.6 & 16.5 \\
\hline Sports & 3 & 5 & 0.6 & 0.5 \\
\hline Trauma & 325 & 3 & 108.3 & 38.0 \\
\hline Total & 749 & $31^{\dagger}$ & 24.1 & 34.3 \\
\hline
\end{tabular}

* The average annual per surgeon reoperation and readmission rate is in the last column; ${ }^{\dagger} 31$ surgeons had complete reoperation and readmission data for the entire 2-year study period. Two surgeons who started and one surgeon who stopped operating during that time period as well as three surgeons who saw patients in the clinic but no longer operated were excluded from this component of the analysis; "no SD data were calculated given there was only one surgeon in the subspecialty.

Table 4. The average annual number of readmission and reoperations per surgeon and their self-reported percentage of complications that are not accounted for by the readmission data because they are managed entirely as an outpatient*

\begin{tabular}{lcc}
\hline Subspecialty & $\begin{array}{l}\text { Mean annual number } \\
\text { of readmissions and } \\
\text { reoperations per surgeon }\end{array}$ & $\begin{array}{l}\text { Mean SD self-reported } \\
\text { parentage of complications } \\
\text { managed as an outpatient }\end{array}$ \\
\hline Arthroplasty & 7.0 & $40.0 \% \pm 44.4 \%$ \\
Foot and ankle & 3.0 & $61.7 \% \pm 49.1 \%$ \\
Hand and upper extremity & 4.8 & $90.0 \% \pm 0.0 \%$ \\
General orthopaedics & 1.0 & $90.0 \%{ }^{\dagger}$ \\
Oncology & 26.5 & $38.3 \% \pm 40.7 \%$ \\
Pediatrics & 2.3 & $85.0 \% \pm 7.1 \%$ \\
Shoulder & 4.0 & $91.7 \% \pm 10.4 \%$ \\
Spine & 13.8 & $18.0 \% \pm 24.6 \%$ \\
Sports & 0.3 & $87.0 \% \pm 9.7 \%$ \\
Trauma & 54.2 & $31.0 \% \pm 20.1 \%$ \\
Total & 12.1 & $59.5 \% \pm 37.1 \%$
\end{tabular}

* This table suggests the magnitude of the true complication rate that readmission and reoperation data likely underestimate; ${ }^{\dagger}$ no SD data were calculated given there was only one surgeon in the subspecialty.

reoperation data likely underestimate the true overall complication rate, surgeons' self-reported percentages of complications treated as an outpatient are listed.

\section{Discussion}

At some level, we all know that human factors play a role in medical incidents just as they do in other industries. The FAA was an early adopter of this concept [4, 5, 7]. The medical field is now catching on, and a cultural shift and a transition to care systems currently are underway. In medicine, investigations into the technical components of a medical incident are becoming secondary to the systems and human factors that contributed to it. One of these human factors is attitude. The FAA believes so strongly that a relationship between attitude and incidents exists, and it requires that all pilots be educated about hazardous attitudes [6]. The FAA has done this on concept validity alone given that a national retrospective and prospective attempt at liking these attitudes to aviation incidents failed [13]. To our knowledge, no publications have investigated 
the impact of surgeons' attitudes on outcomes and no normative data for these attitudes exist in surgeons. In our study, levels of attitudes thought to be hazardous in pilots existed in $30 \%$ of our surgeons (11 of 37) with macho being the most prevalent (24\% of surgeons; nine of 37).

The findings of this study are limited by the lack of accurate error, near miss, incident, and complication data. We suspect that 30-day readmission and reoperation data underestimate the total number of complications. However, patients likely to undergo reoperations are also likely to be readmitted, so some patients may be double-counted using the current method. Although we hope that new safety initiatives and awareness improve self-reported events and accurate incident monitoring, this study is based on a new orthopaedic database, which, although limited, accurately tracks surgeon-specific reoperation and readmission rates. This represents the best currently available data of which we are aware. Anecdotally, reoperation and readmission data are likely to vary substantially by service; this article failed to identify that difference most likely because of having only a 2-year data collection period. A longer collection period would likely reflect the true differences among subspecialties. Additionally, the hazardous attitudes scales are validated in drivers and used in pilot populations, and although they may be valid in surgeons, this has not been tested. Specifically, there are no normative data for the attitudes in surgeons and the modified tools used to measure them have not been rigorously validated. Furthermore, wording of surgeon questions may not be interpreted as neutral and a surgeon wanting to avoid negative responses may elect responses that are more positive. Lastly, $10 \%$ of surgeons (four of 41) who did not respond all were from the same specialty in this sample, and this could have introduced bias.

Additionally, many confounding variables that may influence readmission and reoperation rates were not measured; for example, a complicated infection transferred from an outside hospital. Although these patients do not necessarily represent a complication, they were included in the current method of using the total 30-day readmission and reoperation data. Nevertheless, it is important to consider that few, if any, of the confounding variables are truly independent from the influence of surgeons' attitudes. Surgeon attitudes have a complex interplay with nearly all conceivable confounding variables being that they influence whether a surgeon accepts a complicated patient in transfer, the surgeon's decision to work at a Level I trauma center or a regional referral center, patient selection for elective surgery, surgical decision-making, aggressiveness of rehabilitation, complexity of cases, etc. In truth, the hazardous attitudes identified in this article are likely the confounding factors that go without mention in other research [23].
In our study, macho attitude predicted $19 \%$ of the variance in reoperation and readmission rates for these surgeons over a 2-year period. Looked at another way, the association between surgeons' macho attitudes and readmission and reoperation rates may be able to predict roughly one of every five events. In pilots this attitude was not associated with a history of an accident or the ability to predict future accident, although the research found that pilots who agree with the statements "I am a very careful pilot" and "I am a very cautious pilot" had a lower risk of a future accident [13]. This lack of association between attitude and accident in pilots may be related to differences in the way pilots have reported or recalled accidents before when the National Transportation Safety Board started collecting general aviation accident, the National Transpiration Safety Board's definition of an accident, or simply lack of effect. The last scenario may be the least likely because an analogous population of college-aged drivers, a group with a relatively high accident rate, and drivers with elevated levels of macho attitude had higher rates of accidents, moving violations, driving while under the influence of alcohol, and less seatbelt use [12]. Regardless, in the hospital setting, elevated levels of macho attitude may not be consistent with the routine delivery of safe surgical care in a teamwork setting where human factors and safe systems are the key to success.

This work is a first attempt to look within ourselves at some of the factors and attitudes that may help us become more effective leaders and safer surgeons. This approach brings in knowledge from aviation authorities around the globe, who have taken specific steps to focus on the consumers' reasonable demands for reliability, quality, safety, and cost-effectiveness. Future areas of study on this topic would include the validation of the Modified Surgeon Hazardous Attitude Scale; the collection of normative data for medical students, trainees, and surgeons; and the establishment of a reliable near-miss, incident, error, and outcomes data repository to see if the association found in this small sample has wider applicability.

Acknowledgments Figures created by medical artist Nicole Wolf (NicoleWolfArt.com).

\section{References}

1. Boehm-Davis DA, Amendola KL. Survey of Behavior and Thought Patterns in Transportation. Fairfax, VA, USA: George Mason University Psychology Department; 1993.

2. Booze CF. Epidemiologic investigation of occupation, age, and exposure in general aviation accidents. Aviat Space Environ Med. 1977;48:1081-1091.

3. Buch G, Diehl A. An investigation of the effectiveness of pilot judgment training. Hum Factors. 1984;26:557-564.

4. Diehl AE, Hwoschinsky PV, Lawton RS, Livack GS, eds. Aeronautical Decision Making for Student and Private Pilots. 
DOT/FAA/PM-86/41. Washington, DC, USA: US Department of Transportation-Federal Aviation Administration; 1987.

5. FAA. Advisory Circular: Aeronautical Decision Making. Advisory Circular 60-22. Washington, DC, USA: US Department of Transportation-Federal Aviation Administration; 1991.

6. FAA. Pilot's Handbook of Aeronautical Knowledge. FAA-H8083-25A. Oklahoma City, OK, USA: US Department of Transportation-Federal Aviation Administration-Flight Standards Service; 2003.

7. FAA. Pilot's Handbook of Aeronautical Knowledge. FAA-H8083-25A. Oklahoma City, OK, USA: US Department of Transportation-Federal Aviation Administration-Flight Standards Service; 2008.

8. Fiorillo CD, Tobler PN, Schultz W. Discrete coding of reward probability and uncertainty by dopamine neurons. Science. 2003;299:1898-1902.

9. Gaba DM, Singer SJ, Sinaiko AD, Bowen JD, Ciavarelli AP. Differences in safety climate between hospital personnel and naval aviators. Hum Factors. 2003;45:173-185.

10. Hartmann CW, Rosen AK, Meterko M, Shokeen P, Zhao S, Singer S, Falwell A, Gaba DM. An overview of patient safety climate in the VA. Health Serv Res. 2008;43:1263-1284.

11. Holt RW, Boehm-Davis DA, Amendola KL, Sweeney MM. The Influence of Thought Patterns on Driving Performance. Fairfax, VA, USA: George Mason University; 1994.

12. Holt RW, Boehm-Davis DA, Fitzgerald KA, Matyuf MM, Baughman WA, Littman DC. Behavioral validation of a hazardous thought pattern instrument. Human Factors 35th Annual Meeting. Fairfax, VA, USA: George Mason University Fairfax; 1991:77-81.

13. Hunter DR. Retrospective and prospective validity of aircraft accident risk indicators. Hum Factors. 2001;43:509-518.

14. Hunter DR. Development of an aviation safety locus of control scale. Aviat Space Environ Med. 2002;73:1-5.

15. Hunter DR. Aviation Safety Locus of Control. 2005. Available at: http://www.avhf.com/html/Evaluation/SafetyLOC/TestResultsPage. asp. Accessed October 14, 2009.
16. Hunter DR. Dealing with hazardous attitudes. 2005. Available at: http://www.avhf.com/html/Evaluation/GMasonHazAttitudeScale/ Hazard_Attitude_Training.htm. Accessed October 14, 2009.

17. Hunter DR. Hazardous Attitudes Assessment II: George Mason University Scale (Version 2.0). 2005. Available at: http://www. avhf.com/html/Evaluation/GMasonHazAttitudeScale/GM2.asp. Accessed October 14, 2009.

18. Hunter DR. Measurement of hazardous attitudes among pilots. International Journal of Aviation Psychology. 2005;15:23-43.

19. Jones JW, Wuebker L. Development and validation of the safety locus of control scale. Percept Mot Skill. 1985;61:151-161.

20. Jones JW, Wuebker L. Safety locus of control and employees' accidents. J Bus Psych. 1993;7:449-457.

21. Kaafarani HM, Itani KM, Rosen AK, Zhao S, Hartmann CW, Gaba DM. How does patient safety culture in the operating room and post-anesthesia care unit compare to the rest of the hospital? Am J Surg. 2009;198:70-75.

22. Kadzielski J, McCormick F, Zurakowski D, Herndon JH. Patient safety climate among orthopaedic surgery residents. J Bone Joint Surg Am. 2011;93:1086.

23. Kadzielski J, Vrahas M. A vascular complication of trochantericentry femoral nailing on a fracture table. Am J Orthop (Belle Mead NJ). 2010;39:E64-66.

24. Simpson P, Wiggins M. Attitudes towards unsafe acts in a sample of Australian general aviation pilots. International Journal of Aviation Psychology. 1999;9:337-350.

25. Singer SJ, Gaba DM, Falwell A, Lin S, Hayes J, Baker L. Patient safety climate in 92 US hospitals: differences by work area and discipline. Med Care. 2009;47:23-31.

26. Singer SJ, Gaba DM, Geppert JJ, Sinaiko AD, Howard SK, Park $\mathrm{KC}$. The culture of safety: results of an organization-wide survey in 15 California hospitals. Qual Saf Health Care. 2003;12: $112-118$.

27. Urban RF. Comparative analysis of social, demographic, and flight-related attributes between accident and nonaccident general aviation pilots. Aviat Space Environ Med. 1984;55:308-312.

28. Yeager C, Janos L. Yeager. New York, NY, USA: Bantam; 1985. 